\title{
Skull defect reconstruction based on a new hybrid level set
}

\author{
Ziqun Zhang ${ }^{\mathrm{a}, \mathrm{b}}$, Ran Zhang ${ }^{\mathrm{a}, \mathrm{b}}$ and Zhijian Song ${ }^{\mathrm{a}, \mathrm{b},{ }^{*}}$ \\ ${ }^{a}$ Digital Medical Research Centre, Fudan University, Shanghai, China \\ ${ }^{b}$ Shanghai Key Laboratory of Medical Imaging Computing and Computer Assisted Intervention \\ (MICCAI), Shanghai, China
}

\begin{abstract}
Skull defect reconstruction is an important aspect of surgical repair. Historically, a skull defect prosthesis was created by the mirroring technique, surface fitting, or formed templates. These methods are not based on the anatomy of the individual patient's skull, and therefore, the prosthesis cannot precisely correct the defect. This study presented a new hybrid level set model, taking into account both the global optimization region information and the local accuracy edge information, while avoiding re-initialization during the evolution of the level set function. Based on the new method, a skull defect was reconstructed, and the skull prosthesis was produced by rapid prototyping technology. This resulted in a skull defect prosthesis that well matched the skull defect with excellent individual adaptation.
\end{abstract}

Keywords: Skull defect, image segmentation, level set method, rapid prototyping

\section{Introduction}

Irregularities of the skull due to birth defect, injury, or illness can cause the brain to be without the protection of a complete skull, resulting in the clinical syndrome of skull defects. In order to restore the integrity of the cranial cavity and maintain a stable physiological intracranial pressure, skull reconstruction should be performed in a timely fashion for the overall wellbeing of the patient.

With the development of medical imaging techniques and information technology, a new skull prosthesis shaping method has been developed [1]. The basic idea of this method is to reconstruct a threedimensional model of the skull defect by way of medical image processing techniques by simulating the anatomy of the patient's skull and creating a personalized skull defect repair prosthesis through a rapid prototyping technique. The use of this technology resulted in an accurate match of the prosthesis to the skull defect to achieve an impressive therapeutic effect [2,3].

According to current literature on prosthesis design for skull defects, there are three main methods, the most popular being the mirroring technique [4]. The model of the prosthesis can be duplicated using a geometric axis of symmetry, and although this method has many applications, it is more suitable for the treatment of unilateral skull damage, and less suitable for bilateral skull defects. Because the skull itself is not completely symmetrical, the matching accuracy needs to be improved. The second

${ }^{*}$ Corresponding author: Zhijian Song, Digital Medical Research Centre, Fudan University, Shanghai, China. Tel.: +8621 54237181; Fax:+8621 54237797; E-mail: zjsong@fudan.edu.cn. 
method is based on surface fitting [5], such as a Bezier surface [6] and radial basis function [7], where a fitting surface can be duplicated according to the edges of the defect for an approximate fit. The main limitation of these two methods is that they only provide an approximation based on mathematical applications, without using the actual anatomy of the patient's skull. In order to overcome the limitations of the first two methods, a third method uses a skull template [8], using a deformation algorithm to approximate an outline of the skull defect. However, the template is usually based on data that are taken from the average human skull. The deformation algorithm obtains the final prosthesis based on the anatomical landmarks on the patient's skull and the template images, but this method is not based on the patient's own skull, and it cannot achieve a personalized prosthesis that highly matches the individual's skull defects.

In order to design personalized prostheses that highly match individual skull defects, this study designed a prosthesis according to the information of the patient's skull and brain tissue. The image of the patient's complete skull outline was obtained by use of a new hybrid level set model, and based on edge and region information without re-initialization. The image of the patient's complete skull outline and the original image of the skull defect were calculated by Boolean operations to obtain the skull prosthesis for repair of the defect. This method, with the introduction of the skull and brain tissue information, can design a personalized prosthesis which closely matches the patient's skull defect, and offers a better repair effect for more complex skull defect areas, such as multiple areas of defects, because of the processing advantage of the topology changes in the level set method.

The next section describes a new hybrid level set method based on edge and region information without re-initialization. Section 3 depicts the use of the new hybrid level set model in combination with information of the patient's skull and brain tissue in the prosthesis design for the skull defect repair. Section 4 contains the discussion.

\section{Method}

Rapid prototyping technology was used to produce the prosthesis for the skull defect repair in this study. The operation flow is shown in Figure 1. In step 1, the skull defect information was obtained from the patient's CT scan data. In step 2, the CT data of the skull defect was processed to acquire a
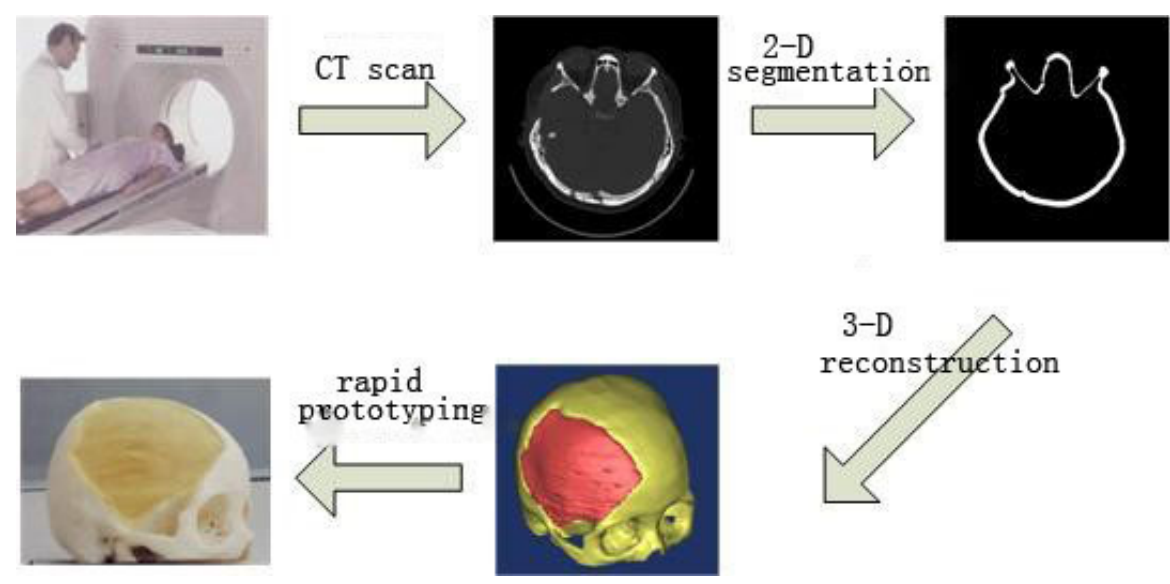

Fig. 1. The operation process of the skull repair system. 
two-dimensional image of a highly-matched personalized prosthesis for the skull defect repair. Step 3 involved a three-dimensional reconstruction of the skull defects for the prosthesis. Step 4 involved the production of the skull defect repair prosthesis by rapid prototyping based on the prosthesis model, which was based on the skull defect data.

\subsection{Image acquisition}

A 44-year-old woman completed a consent form before participating in this study. The skull defects were obtained from the CT data performed with a LightSpeed $16 \mathrm{CT}$ scanner (GE medical systems), matrix size of $512 \times 512$, scanning resolution of $0.449 \times 0.449$, and thickness of $5 \mathrm{~mm}$.

\subsection{New hybrid level set image segmentation}

The image segmentation was the most essential part of the process, which directly affected the accuracy of the subsequent image processing. In this study, a new hybrid level set method was adopted based on edge and region information without re-initialization. This level set method is widely used in image restoration, image enhancement, image segmentation, surface reconstruction, and other fields due to advantages such as topology change ability, easy extension to higher dimension, and solving without parameterization.

\subsubsection{New hybrid level set}

The level set model can be divided into two categories: the edge-based model, represented by the geodesic active contour (GAC) model [9], and the region-based model with Chan-Vese (C-V) model as its typical model [10].

The GAC model primarily uses gradient information to construct an image of the curve evolution process as a result of the final partition.

The GAC energy function is:

$$
\mathrm{E}(\mathrm{c})=\oint_{c} g(C) d s+c \iint_{\operatorname{inside}(C)} g(x, y) d x d y
$$

However, when there is a small gap, the evolving contour curve may penetrate the edge, which means the dynamic profile curve will leak.

To overcome the leakage problem, the $\mathrm{C}-\mathrm{V}$ model was proposed with the use of region information.

$\mathrm{C}-\mathrm{V}$ model energy function is:

$$
\begin{aligned}
& \mathrm{E}\left(c_{1}, c_{2}, C\right)=\mu \oint_{c} d s+\lambda_{1} \iint_{\Omega_{1}}\left(I-c_{1}\right)^{2} d x d y+\lambda_{2} \iint_{\Omega_{2}}\left(I-c_{2}\right)^{2} d x d y \\
& \text { With } c_{1}=\frac{\iint_{\Omega_{1}} I d x d y}{\iint_{\Omega_{1}} d x d y} \quad, \quad c_{2}=\frac{\iint_{\Omega_{2}} I d x d y}{\iint_{\Omega_{2}} d x d y}
\end{aligned}
$$

All the images were divided into two regions, and $c_{1}, c_{2}$ are the current average gray intensities inside and outside the closed contour curve area. 
The $\mathrm{C}-\mathrm{V}$ model has global segmentation capabilities. Minimizing the energy equation makes the global image fit. The $\mathrm{C}-\mathrm{V}$ model is superior to the GAC model in segmenting objects with fuzzy edges and significant noise because it is not based on the image's gray gradient information.

However, the C-V model creates a uniform image intensity distribution, which makes the segmentation imperfect while processing medical images with uneven intensity distribution, due to lack of edge information such as the image's gradient information. It is difficult to obtain $c_{1}, c_{2}$ from medical images with complex backgrounds.

In traditional level set methods, the initial curve of the level set should remain as a signed distance function during its evolution, otherwise peaks or valleys may occur if $|\nabla u| \gg\rangle 1$, and plateaus may occur if $|\nabla u| \ll 1$, resulting in unstable iteration during the numerical calculation. Therefore, re-initialization is required after several times of iteration to make the evolving curve remain the signed distance function consistent with the zero level set. The above process results in a time-consuming curve evolution with low efficiency.

$\mathrm{Li}[11,12]$ added an energy functional in the embedding function $\mathrm{u}$ because in order to completely avoid re-initialization during the level set evolution, the function $u$ is always a signed distance function and satisfies the condition $|\nabla \mathbf{u}|=1$.

An increase in the embedding function $u$ in the energy functional:

$$
E_{i}(\mathrm{u})=\int_{\Omega} \frac{1}{2}(|\nabla u|-1)^{2} d \Omega
$$

Thus, during the curve evolution, if any deviation from $|\nabla \mathrm{u}|=1$ happens, the added energy functional will keep the level set function close to the symbolic distance function, making the evolution of the contour near the zero level set, so there is no need to re-initialize.

A new hybrid level set model based on edge and region information without re-initialization and centered on the advantages of the above model was proposed, and it works well for medical images.

Its energy function is expressed as follows:

$$
\mathrm{E}(u)=-\alpha \iint_{\Omega_{1}}(I-\mu)^{2} H(u) d x d y+\beta \iint_{\Omega} g|\nabla \mathrm{H}(u)| d x d y+\lambda \int_{\Omega} \frac{1}{2}(|\nabla u|-1)^{2} d x d y
$$

where $\alpha, \beta, \lambda$ is a constant predefined as the weight of each term, $I$ is the entire image to be segmented and $g=g(|\nabla \mathrm{I}|)$ is the gray gradient information. Because calculation of the gradient is very sensitive to the noise, the image I $(\mathrm{x}, \mathrm{y})$ needs pre-smoothing. $\mathrm{g}(|\nabla \mathrm{I}|)$ is the image edge indicator, usually a function decreasing with the gray gradient. $\mathrm{g}(|\nabla \mathrm{I}|)$ trends toward zero at the edges where the maximum gradient exists and it is a higher positive value where the gray gradient trends towards zero. The above definition makes the curve evolution rapid in areas of smooth images but slow at the edges of the image.

The first term was based on the region information, where $\mu$ was a pre-defined parameter, the value of which was determined by the gray intensity of the target object. In this study, $\mu$ was defined as a value less than the gray intensity value of the target object, that is, the target object had a higher gray intensity value. If it could not meet this requirement, the image could be preprocessed to satisfy this condition. Minimization of the energy functional pushed the contour curve to the target edge, and this term in the energy function reached the minimum only when the target edge was achieved, resulting in a minimized fitting energy of the image. 
The second term of the function was to ensure that the curve remained positive when the curvature was zero or negative, so that the curve moved toward the target under the force $g(|\nabla \mathrm{I}|)$ and evolution of the curve accelerated.

The third term guaranteed that $|\nabla u|=1$ during the curve evolution, to keep the evolving contour close to the zero level set to avoid re-initialization.

$\delta$ function regularized $\delta_{\varepsilon}$ as an approximation, and the correlated gradient descent flow was:

$$
\frac{\partial u}{\partial t}=\delta_{\varepsilon}(\mathrm{u})\left[\alpha(I-\mu)+\beta \operatorname{div}\left(g \frac{\nabla u}{|\nabla u|}\right)\right]+\lambda \operatorname{div}\left[\left(1-\frac{1}{|\nabla u|}\right) \nabla u\right]
$$

$\delta_{\varepsilon}(\mathrm{u})$ was a positive selection of the derivative of the Heaviside function, and this selection was:

$$
H_{\varepsilon}(z)=\frac{1}{2}\left[1+\frac{2}{\pi} \tan ^{-1}\left(\frac{z}{\varepsilon}\right)\right]
$$

then $\delta_{\varepsilon}(z)=\frac{1}{\pi} \frac{\varepsilon}{\varepsilon^{2}+z^{2}}$, $\varepsilon$ was a parameter used to control $\delta_{\varepsilon}(z)$ of the effective width, with high stability, adopting a large time step.

\subsubsection{Image segmentation}

The new hybrid level set method of image segmentation obtained the information of the outermost layer of the skull by an evolutionary convergence of the selected initial contour image. The information of the skull and the brain tissue was thus obtained after the removal of the unnecessary areas and redundant information.

The new hybrid level set method obtained information of the skull and brain tissue, as shown in Figure 2, where (a) shows the initial contour selected by the level set; (b) shows the iteration result after the level set evolved 120 times; (c) shows the original CT image; (d) shows the image after the removal of redundant information.

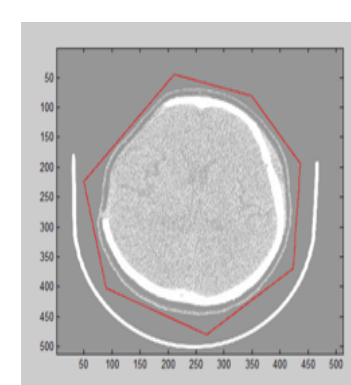

(a)



(b)



(c) (d)

Fig. 2. Image segmentation process. 


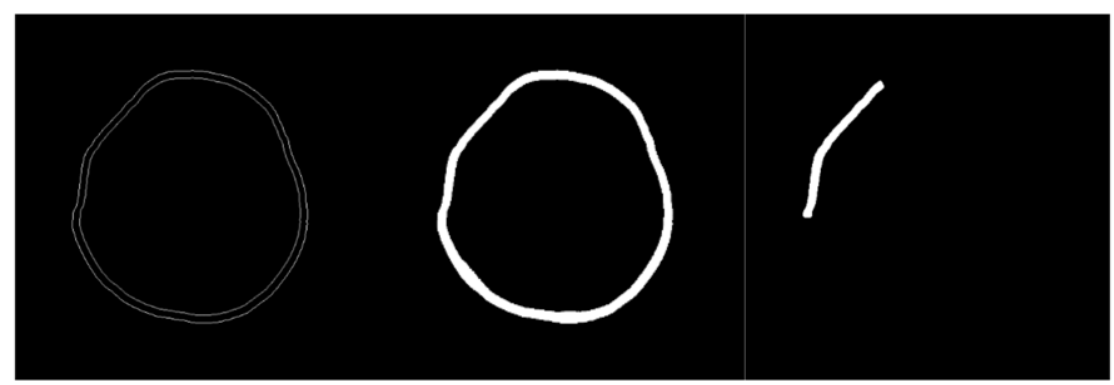

(a)

(b)

(c)

Fig. 3. Results of a complete skull and skull defect outline: (a) Complete skull, inside and outside contour; (b) Complete skull outline; (c) Skull defect outline.

Only the skull and brain tissue information remained in the image, with removal of the redundant information and interference. Thus, the image was ready for the segmentation to obtain the inner and outer contours.

The processed image was then segmented by the new hybrid level set method according to the skull and brain tissue information to obtain the complete inner contour of the skull. The initial curve was inside the skull and the complete outer contour of the intact skull was also obtained. The contour conformed closely with the intact part of the skull after debugging and validating. Thus, the complete skull image was obtained. The obtained complete skull image and the defect area of the original skull image were Boolean calculated to finally acquire the information of the skull defect area, as shown in Figure 3.

\subsection{Three-dimensional reconstruction and rapid prototyping}

The result data was processed by the threshold selection, regional growth, and other operations to obtain the three-dimensional data of the skull defect contour. The above three-dimensional data of the prosthesis for the skull defect was input to the 3D printer software in stl file format to obtain the ultimate prosthesis model for the repair of the skull defect.

\section{Results}

\subsection{Results obtained from the contour of the skull defect area}

The image was segmented by the new hybrid level set method and the inner skull contour information was obtained after evolution and iteration. The outer skull contour was obtained by ratio expansion of the inner contour, and thus, the complete skull image was established. The complete skull image was Boolean calculated with the original image of the defected skull, followed by corrosion and expansion to obtain the image of the prosthesis for the defect area repair, as shown in Figure 4. 


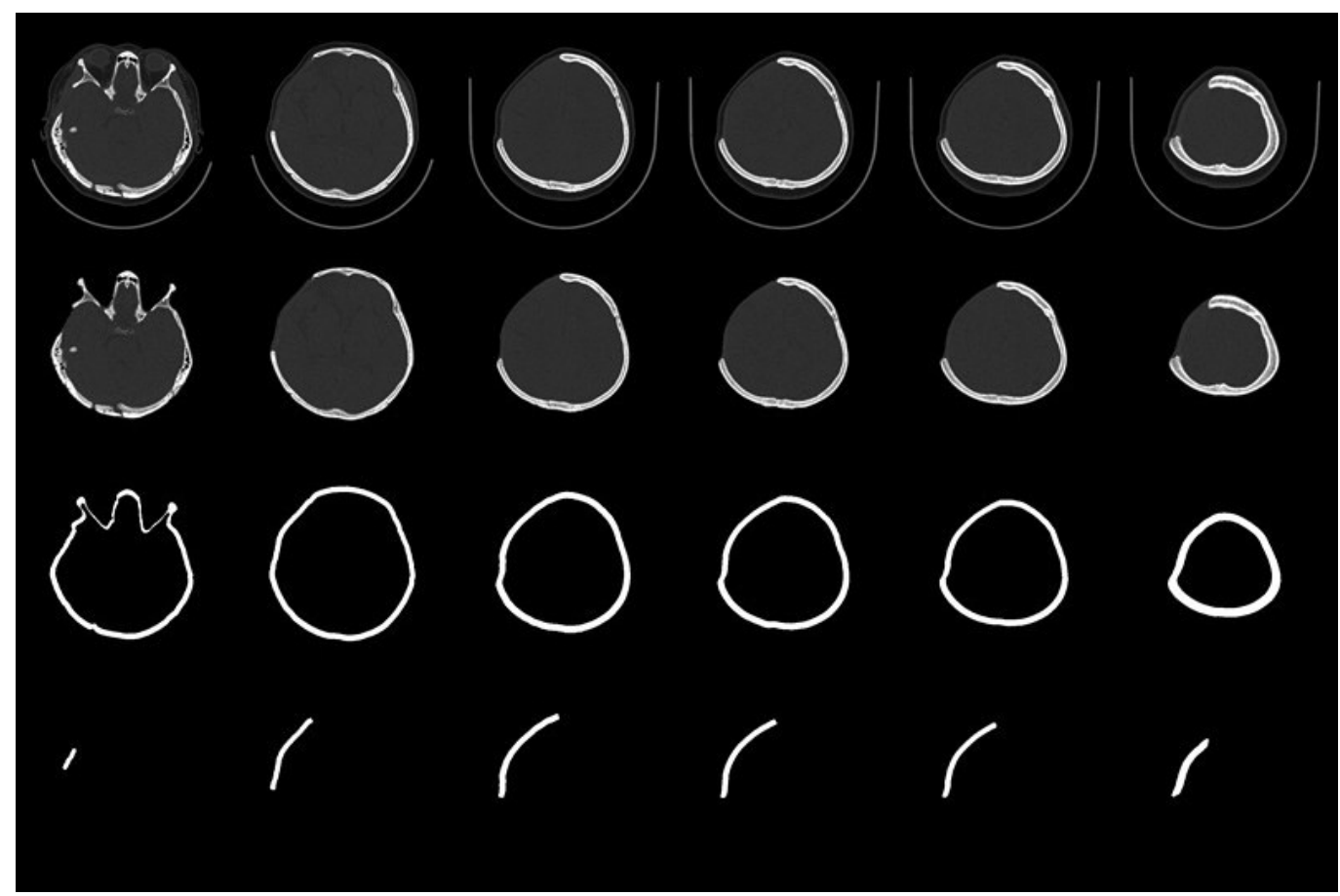

Fig. 4. Complete skull and skull defect outline results. The first line shows the original CT images, the second line shows the images after removal of redundant information, the third line shows the images of a complete skull, and the fourth line shows the images of the skull defect.

\subsection{Personalized molding manufacture of skull prosthesis}

The skull CT image sequences were processed. The two-dimensional CT image sequences of the defected skull and two-dimensional image sequences of the prosthesis for skull defect repair were three-dimensionally reconstructed to obtain a high quality surface model. After verifying that the prosthesis effectively matched the original skull defect, the results of the surface data were output in RP format, as shown in Figure 5. Finally, a personalized prosthesis that well matched the skull defect was produced by rapid prototyping. Hence, restoration of the patient's skull was accomplished, as shown in Figure 6.

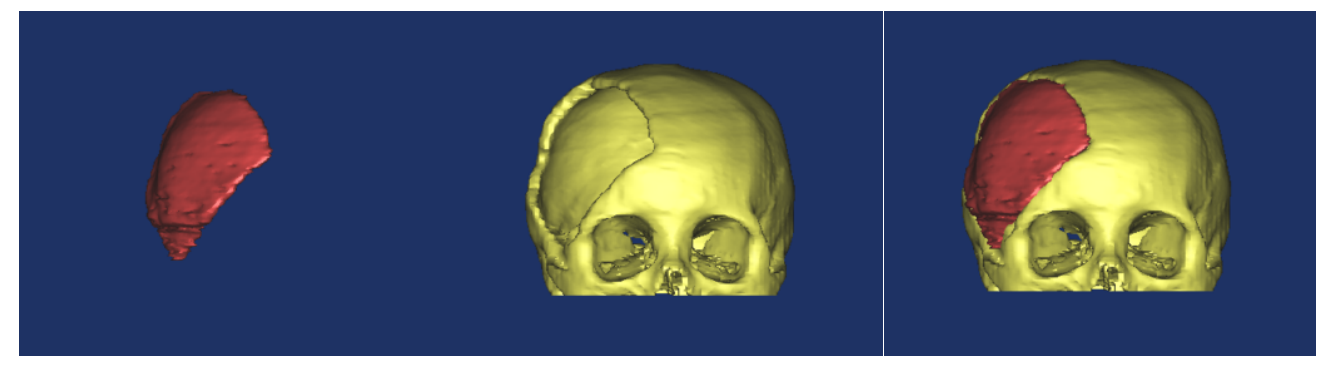

(a)

(b)

(c)

Fig. 5. Three-dimensional model of prosthesis for skull defect repair: (a) 3D model of prosthesis for skull defect repair; (b) Patient's original skull 3D model; (c) Repaired skull 3D model. 




(a)

(b)

Fig. 6. Results of rapid prototyping: (a) Defected skull model; (b) Adaptation result of the personalized prosthesis.

\section{Discussion}

Skull defect reconstruction based on digital and rapid prototyping technology was highly praised by medical workers for its personalization, accuracy, and effectiveness. The model design of the prosthesis for skull defect repair directly determined the therapeutic effect of the skull defect restoration. In this study, a method for the prosthesis design was proposed by establishing a model according to the image information of the patient's own skull and brain tissue. Based on the image information of the patient's skull and brain tissue, this method extracted an integral contour of the patient's skull quickly and accurately by use of level set algorithm, which is capable of handling images with complex shape and changeable topology. This method differs from the method based on mirroring reconstruction, which assumes that information on the contralateral side of the skull is available. However, if the damage is over the midline of the skull or the image information of the contralateral side is incomplete, it is difficult to accomplish repair of skull defects by this method. Although the surface fitting method can be used to reconstruct prosthesis for defects of an asymmetrical skull, such reconstruction is a rough estimation based on the mathematical model rather than restoration based on image information of the patient's individual anatomy.

In this study, the skull prosthesis established by use of image data based on the patient's anatomy is closer to the actual shape of the patient's skull than that established by use of a template based on average data of the skull images.

This study proposed a new hybrid level set model for restoration of skull defects and tested this method with CT image data. Patients enrolled in this study all suffered from unusually large skull defects, which made it more difficult to design the prostheses. In this study, the prosthesis models were established by the proposed method, and the results showed that the prostheses prefabricated by rapid prototyping techniques based on the models established by introduction of brain tissue information well matched the skull defects. Such a method can provide highly personalized prostheses for skull defect repairs, exempting the necessity of multiple operations, and assuring a more natural looking reconstruction.

\section{Acknowledgement}

This study was supported by the National Science and Technology Support Program of China (2012BAI14B05). 


\section{References}

[1] L.C. Hieu, N. Zlatov, J. Vander Sloten et al., Medical rapid prototyping applications and methods, Assembly Automation 25 (2005), 284-292.

[2] Y.L. Liao, C.F. Lu, Y.N. Sun et al., Three-dimensional reconstruction of cranial defect using active contour model and image registration, Med. Biol. Eng. 49 (2011), 203-211.

[3] Y. Zhao, W. Li, M. Manhein et al., An automatic assembly and completion framework for fragmented skulls, IEEE International Conference on Computer Vision, 2011, 2532-2539.

[4] E. Maravelakis, K. David, A. Antoniadis et al., Reverse engineering techniques for cranioplasty: A case study, J. Med. Eng. Technol. 32 (2008), 115-121.

[5] G.Y. Huang and L.J. Shan, Research on the digital design and manufacture of titanium alloy skull repair prosthesis, The 5th International Conference on Bioinformatics and Biomedical Engineering, 2011.

[6] C.S. Chong, H.P. Lee and A.S. Kumar, Automatic hole repairing for cranioplasty using Bezier surface approximation, J. Craniofac. Surg. 17 (2006), 344-352.

[7] J.C. Carr, W.R. Fright and R.K. Beatson, Surface interpolation with radial basis functions for medical imaging, IEEE Trans. Med. Imaging 16 (1997), 96-107.

[8] D. Dean and K.J. Min, Deformable templates for preoperative computer-aided design and fabrication of large cranial implants, Int. Congr. Ser. 1256 (2006), 710-715.

[9] V. Caselles, T. Catte, T. Coll et al., A geometric model for active contours in image processing, Numeric Math 66 (1993), 1-31.

[10] T.F. Chan and L.A. Vese, Active contour without edges, IEEE Trans. Image Proeess 10 (2001), 266-277.

[11] C.M. Li, C.Y. Xu, C.F. Gui et al., Level set evolution without re-initialization: A new variational formulation, IEEE Computer Society Conference on Computer Vision and Pattern Recognition, 2005, 430-436.

[12] C.M. Li, C.Y. Xu, C.F. Gui et al., Distance regularized level set evolution and its application to image segmentation, IEEE Transactions on Image Processing 19 (2010), 3243-3254. 